\title{
Les consultants français en management et le « Balanced Scorecard » (BSC) : enquête sur les pratiques d'un modèle de gestion nord-américain en France
}

\author{
Jonathan Dreyfus-Schmidt, MBA, et Jacques Grisé, Ph. D., F.Adm.A. \\ Université Laval
}

\section{Introduction}

Il est légitime de se demander si le modèle du BSC est adapté au contexte socioorganisationnel français, car il est beaucoup moins utilisé en France que dans d'autres pays, particulièrement aux États-Unis ${ }^{1}$. On estime qu'environ $60 \%$ des firmes américaines du Fortune 500 utilisent un système de BSC. Le BSC a aussi connu du succès en Finlande ${ }^{2}$ et en Scandinavie $^{3}$. Une étude a montré que $41 \%$ des entreprises françaises connaissent le BSC et que seulement $3 \%$ d'entre elles pensent le mettre en œuvre. On peut à juste titre se demander si la dimension idéologique du BSC est compatible avec l'idéologie française de la gestion ${ }^{4}$.

Les consultants en management français constituent une interface entre les organisations françaises et le domaine du management. Ils peuvent jouer un rôle particulier dans la propagation des idées relatives au management auprès des dirigeants français.

\section{Les consultants en management français constituent une interface entre les organisations françaises et le domaine du management. Ils peuvent jouer un rôle particulier dans la propagation des idées relatives au management auprès des dirigeants français.}

Les résultats de l'enquête témoignent d'une grande diversité dans les profils des utilisa- teurs du BSC, leur compréhension du modèle et leur utilisation du BSC. L'enquête permet d'avancer quelques éléments de réponses aux quatre questions ci-dessous.

- Quels cabinets de conseil en management utilisent le BSC? Même si 44 \% des cabinets de conseil en management ont déjà mis en œuvre des BSC, ce sont surtout les cabinets de plus de 10 millions d'euros et de plus de 50 consultants qui l'utilisent.

- Comment les fondements du BSC sont-ils compris par les consultants? Les consultants ont une définition assez hétéroclite du BSC, mais ses grands principes sont généralement bien compris.

- Comment se déroule la mise en cuvre du BSC par les consultants? La direction générale est toujours à l'origine du projet BSC. Les consultants utilisent une démarche de mise en œuvre participative et ils n'utilisent pas de logiciel. Ils jugent que le processus de diffusion en cascade est indissociablement lié au $\mathrm{BSC}$, mais qu'il est difficile à mettre en œuvre. Les phases de mise en œuvre du BSC sont utilisées avec souplesse. L'utilisation du BSC semble apporter des résultats, mais ceuxci sont plutôt qualitatifs et relativement difficiles à mesurer.

- Pourquoi le BSC est-il moins utilisé en France que dans d'autres pays? Les deux 
tiers des consultants expliquent la sousutilisation du BSC en France par des différences culturelles sur les pratiques de gestion avec les pays anglo-saxons. Parmi les différences culturelles observées, on peut noter la culture des résultats, le rôle de l'État dans l'économie et le moindre recours au conseil externe en France. Les consultants sont plutôt optimistes sur le développement du BSC en France. Ils remarquent toutefois qu'il doit être adapté au contexte culturel français et aux besoins des entreprises pour être efficace.

\section{Méthodologie de l'étude}

La stratégie de recherche de cette étude est descriptive. L'enquête a pris la forme d'entrevues avec pour complément un guide d'entretien semi-directif.

La population d'étude a été définie à partir d'un annuaire des sociétés de conseil en management qui identifie les 100 premiers cabinets présents sur le marché français ${ }^{5}$. Au total, 106 sociétés ont été contactées. Cellesci emploient 20213 consultants, soit $85 \%$ du total des sociétés de conseil en management en 2002. Elles représentent un chiffre d'affaires cumulé de 3,8 milliards d'euros en 2002 sur un total de 4,2 milliards pour toute la profession du conseil en management en France. Ces données sont issues du rapport annuel 2002 de Syntec Conseil Management. Ce syndicat est indépendant et représente les professionnels du conseil en management en France.

Sur les 106 cabinets de conseil contactés, 47 possèdent une expérience de mise en œuvre de BSC, et parmi ceux-ci, 17 ont été interviewés. Chaque interview a duré en moyenne 25 minutes. Ces 17 entreprises représentent un chiffre d'affaires cumulé de 1,3 milliard d'euros et 6500 consultants, soit respectivement $43 \%$ et $39 \%$ du total cumulé des 47 cabinets ayant une expertise BSC.

\section{Quels sont les cabinets de conseil en management qui utilisent le BSC?}

\section{Profil des cabinets de conseil en management}

Les 106 cabinets de conseil interrogés ont différents niveaux d'expérience avec le BSC. Dans $44 \%$ des cas, les cabinets interrogés possèdent au moins une expérience de mise en place du BSC. Ces entreprises comptent au moins un consultant spécialiste de la question. Dans $21 \%$ des cas, les cabinets connaissent le BSC, mais ils n'ont pas d'expérience particulière dans ce domaine et n'ont aucun consultant spécialiste de la question. Dans $34 \%$ des cas, les cabinets ne connaissent pas et n'ont jamais utilisé de BSC.

En incluant les cabinets de conseil qui connaissent le BSC sans toutefois l'utiliser, le « taux de notoriété » du BSC au sein des cabinets de conseil en management français s'élève à $65 \%$. Avec leur formation en management ainsi que leur accès aux revues spécialisées, il est surprenant que le tiers des cabinets de conseil interrogés n'ait jamais entendu parler du BSC.

Ce ne sont pas tous les cabinets de conseil qui possèdent une expertise en BSC. Alors que les cabinets de plus de dix millions d'euros de chiffre d'affaires totalisent $48 \%$ de la population de l'étude, ils représentent $69 \%$ des cabinets de conseil possédant une expertise en BSC. Alors que les cabinets de conseil de plus de 50 consultants ne représentent que $12 \%$ de la population totale de l'étude, ils forment $75 \%$ des cabinets de conseil possédant une expertise en BSC. 
En bref, le BSC est aussi bien utilisé par les petits cabinets de conseil que par les grands. Cependant, beaucoup d'entreprises moyennes (de 5 à 50 millions d'euros de chiffre d'affaires et de 10 à 100 consultants) n'ont pas d'expertise alors que presque tous les plus grands cabinets possèdent une expertise.

\section{Les spécialistes français du BSC sont tous} des consultants expérimentés. La moitié d'entre eux possède plus de dix années d'expérience dans le conseil. Ils ont en majorité une formation de type-école de commerce (44\%), universitaire ( $24 \%)$, MBA (16\%) ou école d'ingénieur (16\%).

\section{Profil des consultants experts en BSC}

Les spécialistes français du BSC sont tous des consultants expérimentés. La moitié d'entre eux possède plus de dix années d'expérience dans le conseil. Ils ont en majorité une formation de type-école de commerce (44\%), universitaire $(24 \%)$, MBA $(16 \%)$ ou école d'ingénieur (16\%). La lecture de livres, en particulier ceux de Kaplan et Norton, est pour la moitié des consultants leur seule formation sur le BSC. L'autre moitié déclare avoir reçu une formation spécifique au BSC (formation interentreprise, formation interne ou externe). La majorité des consultants cumule une expérience de un à trois ans dans la mise en œuvre du BSC (59\%), les autres ont plus de trois ans d'expérience (41\%).

\section{Comment les fondements du BSC sont-ils compris par les consultants?}

Les consultants définissent très différemment le BSC, mais ses grands principes sont généralement bien compris

Comme le montre le tableau 1, les consultants définissent majoritairement le BSC comme un outil d'alignement stratégique et de mise en cohérence entre la stratégie et les actions des employés. Avec environ deux tiers de réponse, cette idée est celle qui est la mieux associée au BSC même si elle est exprimée différemment selon les consultants. Pour les uns, le BSC est « un outil permettant de créer un consensus autour de la stratégie d'une entreprise et de la décliner en objectifs opérationnels », pour les autres, «c'est un outil de verrouillage qui assure une cohérence dans l'entreprise et aligne les actions des employés sur la stratégie $»$.

\section{Le BSC est un « outil qui permet de rendre} responsable individuellement et collectivement chaque manager de la performance de l'entreprise ».
Les consultants associent à $41 \%$ le BSC avec l'idée d'un système de pilotage ou de gestion stratégique. Le BSC est un « outil qui permet de rendre responsable individuellement et collectivement chaque manager de la performance de l'entreprise $»$.

Les consultants définissent majoritairement le BSC comme un outil d'alignement stratégique et de mise en cohérence entre la stratégie et les actions des employés.

Les consultants font une différence entre le BSC et les traditionnels tableaux de bord. Comme l'illustrent certains témoignages, « le BSC est plus dynamique qu'un simple outil de mesure de la performance. Il relève plus de l'outil d'aide au pilotage stratégique que du simple instrument de mesure». Le BSC « aide à gérer et surtout anticiper. Contrairement aux simples instruments de mesures de la performance assez passifs, le BSC est proactif et donne une vision plus anticipée de la performance future de l'entreprise ». 


\section{Tableau 1 : Définition du Balanced Scorecard par les consultants français en management}

\begin{tabular}{|lc|}
\hline \hline Définition du Balanced Scorecard & $\%$ \\
\hline 1. Idée d'alignement stratégique et de cohérence entre stratégies de l'entreprise & $65 \%$ \\
$\quad$ et action des employés. & \\
2. Idée de pilotage stratégique ou de gestion stratégique. & $41 \%$ \\
3. Idée de mesure de la performance. & $29 \%$ \\
4. Idée de communication de la stratégie et de recherche de consensus. & $29 \%$ \\
5. Idée d'équilibre dans la mesure de la performance. & $24 \%$ \\
\hline \hline
\end{tabular}

\section{Le BSC « aide à gérer et surtout anticiper. Contrairement aux simples instruments de mesures de la performance assez passifs, le BSC est proactif et donne une vision plus anticipée de la performance future de l'entreprise ".}

Cités à égalité avec $29 \%$ de réponses chacun, les termes «mesure de la performance» et « communication de la stratégie et recherche de consensus» sont tous les deux bien associés au BSC.

Seulement un quart des répondants pense au principe d'équilibre des indicateurs, et évoque spontanément les quatre axes du modèle (Financier, Client, Processus internes et apprentissage organisationnel). En résumé, les consultants ont chacun leur propre définition $\mathrm{du}$ BSC, mais les principaux principes et éléments à prendre en compte sont généralement bien compris.

\section{Les fonctions du BSC sont diversement appréciées par les consultants}

Dans leur ouvrage de référence publié en France en 1998, Kaplan et Norton ${ }^{6}$ identifient sept fonctions principales du BSC. Les consultants n'accordent pas la même importance à toutes les fonctions qui sont présentées dans le tableau 2.

D'autres fonctions non proposées ont été citées comme la gestion du changement et la gestion de projets. On remarque aussi que le BSC n'est pas utilisé en France pour définir la rémunération. Bien qu'il puisse être utilisé pour définir la rémunération des dirigeants, le BSC ne l'est pas du tout pour celle des employés. Un consultant remarque qu'« on

\section{Tableau 2 : Les principales fonctions du Balanced Scorecard}

selon les consultants français en management

\begin{tabular}{|lc|}
\hline \hline Fonctions du Balanced Scorecard & $\mathbf{\%}$ \\
\hline 1. Faire concorder les objectifs des unités et des individus avec la stratégie de l'entreprise. & $28 \%$ \\
2. Clarifier la stratégie et réunir un consensus autour d'elle. & $18 \%$ \\
3. Relier les intentions stratégiques aux objectifs longs termes et aux budgets annuels. & $18 \%$ \\
4. Faire connaître la stratégie à toute l'entreprise. & $16 \%$ \\
5. Assurer le retour d'expérience sur la stratégie et l'affiner progressivement. & $10 \%$ \\
6. Procéder régulièrement et de façon systématique à des bilans stratégiques. & $8 \%$ \\
7. Identifier et harmoniser les initiatives stratégiques. & $2 \%$ \\
\hline \hline
\end{tabular}


n'est pas allé jusqu'au bout de la démarche BSC sur ce point, car la rémunération suit une logique plus ‘statutaire' en France ».

Interrogés spécifiquement sur le lien entre stratégies et BSC, la moitié des consultants estime que celui-ci "permet d'aligner les actions des employés sur la stratégie», et «constitue un levier pour mettre toute l'entreprise en relation avec la stratégie ». Cette idée est très clairement associée au BSC puisqu'elle est déjà la plus citée pour définir le BSC.

Pour la plupart des consultants, le BSC aide les dirigeants à organiser le fruit de leurs réflexions sur leur stratégie, mais il ne permet en aucun cas de «créer» une stratégie. Le BSC permet plutôt de la « formaliser ».
Évaluer la compréhension des fondements du BSC par les consultants permet de mieux comprendre comment ils le mettent en place. La diversité des réponses sur les définitions et les fonctions du BSC laisse présager de grandes différences dans les utilisations de cet outil par les consultants.

Pour la plupart des consultants, le BSC aide les dirigeants à organiser le fruit de leurs réflexions sur leur stratégie, mais il ne permet en aucun cas de " créer» une stratégie. Le BSC permet plutôt de la « formaliser ».

\section{Comment se déroule la mise en place du BSC par les consultants?}

\section{La direction générale est toujours à l'origine du projet BSC}

L'origine de la mise en œuvre d'un BSC vient toujours «d'une équipe de direction, peu importe son niveau, du moment qu'elle soit autonome et dirige son budget ». Un consultant note que la démarche est instaurée plus souvent par la «direction de la qualité » que par la «direction financière». Un autre souligne que le BSC peut être mis en œuvre dans des «business-units» ou uniquement dans des unités structurées sur la base des fonctions principales de l'entreprise.

Très peu de consultants ont affirmé recevoir des demandes de mise en place de BSC de la part des dirigeants français. Le plus souvent, les consultants proposent une intervention BSC après une analyse des besoins de l'entreprise et, dans tous les cas, celle-ci s'adresse à la direction de l'entreprise cliente.

\section{Les phases de mise en ouvre du BSC sont adaptées par les consultants français}

Comme le montre le tableau 3, presque la moitié des consultants estime qu'il faut inclure un travail préliminaire sur la stratégie de leur client avant de procéder à une intervention. La définition d'indicateurs et facteurs clefs de succès $(35 \%)$ et la définition d'objectifs ou de plans d'action (29\%) sont aussi des éléments souvent cités.

Environ $40 \%$ des consultants ont déclaré utiliser la méthode de Kaplan et Norton, et ces derniers ont reconnu l'adapter aux besoins de leurs clients. Beaucoup de consultants utilisent une démarche qui leur est propre. Ils n'hésitent pas à modifier le modèle du BSC qui est qualifié de «trop lourd» par un tiers des répondants interviewés.

Un quart des consultants insiste sur le fait qu'il faut construire le BSC en collaboration avec les opérationnels. Il faut "vendre le 
Tableau 3 : Principales phases de mise en œuvre du Balanced Scorecard selon les consultants français en management

\begin{tabular}{|lll|}
\hline \hline \multicolumn{2}{l}{ Phases de mise en ouvre } & $\%$ \\
\hline 1. & Travail préliminaire sur la stratégie avant la mise en œuvre du BSC. & $47 \%$ \\
\hline 2. & Utilisation des phases de Kaplan et Norton en les adaptant en fonction du client. & $41 \%$ \\
\hline 3. & Définition d'indicateurs et des facteurs clefs de succès. & $35 \%$ \\
\hline 4. & Définition d'objectifs et de plans d'action. & $29 \%$ \\
\hline 5. & Validation et association avec les opérationnels. & $24 \%$ \\
\hline \hline
\end{tabular}

projet auprès des employés » et « associer les utilisateurs dès les phases amont du projet». Un consultant remarque aussi que « la mise en œuvre du BSC doit être incrémentale. Son déploiement étant assez lourd, il est préférable de commencer à l'introduire dans un business-unit » ou un « site-pilote ».

\section{Un quart des consultants insiste sur le fait qu'il faut construire le BSC en} collaboration avec les opérationnels. Il faut « vendre le projet auprès des employés » et « associer les utilisateurs dès les phases amont du projet ».

En synthèse, on peut noter qu'il existe beaucoup de démarches différentes lors de la mise en place d'un BSC par des consultants en France. Les consultants n'hésitent pas à adapter le modèle aux besoins de leurs clients et à utiliser de manière souple la méthodologie du BSC.

Pour une majorité de consultants, l'intervention BSC dure en moyenne de trois à six mois

Plus de la moitié (53\%) des consultants estime qu'il faut en moyenne de trois à six mois pour réaliser une intervention BSC. Un quart des consultants pense qu'il faut en moyenne six mois à un an pour mettre en œuvre un BSC et $18 \%$, plus d'un an. Même si tous les consultants soulignent que la durée de l'intervention dépend fortement des besoins du client, de son environnement et de sa culture d'entreprise, on remarque que les consultants français prennent plus de temps que les entreprises américaines. En effet, aux États-Unis, la plupart des entreprises ont implanté leurs systèmes de tableaux de bord en moins de six mois, et $31 \%$ l'ont fait en moins de trois mois ${ }^{7}$.

Le temps nécessaire à la mise en œuvre du BSC n'est pas suffisant. Il faut également du temps pour que le BSC soit accepté par ses utilisateurs et l'entreprise en général. Cette phase de «digestion » du BSC prend plus de temps que la phase de mise en œuvre. Près de $70 \%$ des consultants estiment qu'il faut au moins six mois, un quart pense même qu'il faut plus d'un an.

\section{Les consultants utilisent une démarche de mise en ouvre participative}

Les démarches «Top-Down» et «participative » sont respectivement utilisées par les consultants français à $39 \%$ et $43 \%$. Le « Bottomup » n'est utilisé que par $17 \%$ des consultants, mais, dans tous les cas, il est utilisé en parallèle d'une démarche "Top-Down ». Un consultant explique sur ce point que la démarche doit «être Top-Down dans un premier temps avant d'être Bottom-up pour faire remonter les informations opération- 
nelles. En définitive, la démarche devient participative ». Comme le souligne ce consultant, la démarche participative permet «d'échanger des idées sur la stratégie de l'entreprise, elle favorise le partage d'informations $»$.

Le BSC est, en théorie, un système " TopDown » qui distingue la phase d'élaboration de la stratégie de la phase d'application. La démarche participative facilite la recherche de consensus et de cohérence autour de la stratégie en associant à la fois les opérationnels et la direction dans la construction du BSC. Elle est plus utilisée par les consultants français et semble mieux adaptée au contexte des entreprises françaises.

Le BSC est, en théorie, un système « Top-Down » qui distingue la phase d'élaboration de la stratégie de la phase d'application. La démarche participative facilite la recherche de consensus.

Le processus de diffusion en cascade est indissociable du BSC, mais il est difficile à mettre en œuvre

À trois exceptions près, les consultants ont tous affirmé « cascader » le BSC, c'est-à-dire le diffuser à plusieurs niveaux dans l'entreprise. La grande majorité d'entre eux comprend que le BSC doit «se décliner» dans l'entreprise pour relier son sommet stratégique à sa base opérationnelle. Les consultants qui ne « cascadent» pas le BSC le réservent à la direction générale. Ils n'utilisent le modèle que pour ses fonctions de clarification et de formalisation de la stratégie.

Même s'il est fortement associé à la démarche $\mathrm{BSC}$, le processus de cascade est jugé difficile à mettre en œuvre. Selon un témoignage, le BSC est « un outil qui intéresse les dirigeants, mais ceux-ci ont du mal à le diffuser en cascade. Les leviers sont difficilement partageables avec l'ensemble des employés (notamment l'axe financier)». Même si «le processus de cascade permet d'instaurer un dialogue positif au sein de l'entreprise, son déploiement est lourd et long à mettre en place» remarque un consultant. Plus l'entreprise est grande, plus le processus de cascade est difficile à mettre en œuvre en France.

\section{Les trois quarts des consultants n'utilisent pas de logiciel}

Le degré d'utilisation de logiciels semble lié à la taille du cabinet. Ce ne sont que les plus grands cabinets de conseil qui déclarent utiliser des logiciels. SAP Strategic Entreprise Management et Hyperion Performance Scorecard sont les deux seules offres logicielles citées par les consultants qui utilisent un logiciel $(24 \%$ du total). Les solutions informatiques intégrées ne sont pas utilisées, car elles sont jugées trop coûteuses et pas assez souples. Elles ne sont pas non plus adaptées à une mise en œuvre localisée du BSC (Business Unit, division ou fonction d'une entreprise). Un consultant remarque que l'offre logicielle peut devenir une contrainte importante pour le client, car elle est souvent assez « coûteuse, longue et difficile à mettre en place ». Seules les plus grandes entreprises françaises peuvent se permettre de mettre en place des BSC informatisés.

\section{La faible utilisation de logiciels par les consultants peut s'expliquer par une faible utilisation des progiciels de gestion par les entreprises françaises.}

La faible utilisation de logiciels par les consultants peut s'expliquer par une faible utilisation des progiciels de gestion par les entreprises françaises. Comme le fait en effet remarquer un consultant, « aux États-Unis, les 
outils de gestion s'achètent comme de quelconques produits. Ce n'est pas le cas en France ». Les entreprises françaises développent plus souvent leurs outils de gestion à l'interne et elles ont peu recours aux systèmes de gestion "clef en main " vendus par de nombreux prestataires de services.

Le degré d'utilisation de progiciels de gestion avec des fonctionnalités BSC est nettement moins important en France qu'aux États-Unis. Une étude menée auprès des entreprises américaines a montré que $« 70 \%$ des entreprises qui possèdent un système de tableau de bord font appel à un certain type de logiciel afin de simplifier ces tâches $»^{7}$. À titre de comparaison, le taux d'utilisation d'un progiciel ERP (Enterprise Resource Planning) par les entreprises françaises s'élève à $30 \%, 25 \%$ et $50 \%$ respectivement pour le secteur des services, du commerce et de l'industrie manufacturière (Sessi et Scees, enquêtes 2002 « TIC et le commerce électronique »).

Le degré d'utilisation de progiciels de gestion avec des fonctionnalités BSC est nettement moins important en France qu'aux États-Unis.

\section{La principale contrainte du BSC aux yeux des clients}

Même si certains consultants estiment que l'on peut mesurer les résultats du BSC, $29 \%$ d'entre eux pensent qu'il s'agit de la principale lacune aux yeux de leurs clients. Le coût du projet BSC $(10 \%)$, le temps nécessaire à sa mise en œuvre $(10 \%)$ et l'implication du management $(10 \%)$ sont des contraintes peu évoquées.

D'autres contraintes ont été citées par un quart des consultants. Par exemple, « la disponibilité réduite des équipes des consultants et des participants à un projet BSC peut », selon un consultant, " ralentir son processus de mise en œuvre ». Un consultant confie aussi que «de nombreux clients ne comprennent le BSC qu'en complément des tableaux de bord existant dans l'entreprise. Ils n'ont pas une logique de remplacement, ce qui complexifie le BSC aux yeux du client ».

\section{Presque la moitié des consultants affirme que le BSC permet d'améliorer \\ l'alignement stratégique et la mise en \\ cohérence de la stratégie et des actions des employés.}

Les avis sur les résistances au BSC sont contradictoires. Plusieurs consultants ont rappelé avec raison que le BSC est avant tout un outil. Pour eux, il n'y a pas de contrainte intrinsèque au modèle. Tout dépend $d u$ contexte d'utilisation et de son adéquation aux besoins du client.

Le BSC apporte des résultats tangibles, mais ceux-ci sont plutôt qualitatifs et relativement difficiles à mesurer

La mise en œuvre d'un BSC conduit à des résultats très différents selon les entreprises. Comme le montre le tableau 4, presque la moitié des consultants affirme que le BSC permet d'améliorer l'alignement stratégique et la mise en cohérence de la stratégie et des actions des employés.

Plus d'un tiers des répondants affirme que c'est en matière de la clarification de la stratégie et de la réalisation d'un consensus que le BSC apporte les meilleurs résultats. Pour un consultant, le BSC permet en effet de «clarifier les idées et la stratégie dans l'entreprise. Il montre le niveau d'implication des dirigeants et des employés dans la stratégie de l'entreprise. Il permet d'obtenir consensus et responsabilisation ». 


\section{Tableau 4 : Principaux résultats apportés par le Balanced Scorecard}

selon les consultants français en management

\begin{tabular}{|c|c|}
\hline Résultats & $\%$ \\
\hline $\begin{array}{l}\text { 1. Alignement stratégique et mise en cohérence de la stratégie et des actions des } \\
\text { employés. }\end{array}$ & $47 \%$ \\
\hline 2. Clarification de la stratégie et établissement d'un consensus. & $35 \%$ \\
\hline 3. Difficile de mesurer les résultats et cela dépend des entreprises. & $29 \%$ \\
\hline 4. Fixation d'objectifs et de plans d'action. & $24 \%$ \\
\hline 5. Amélioration globale de la performance de l'entreprise et atteinte d'objectifs chiffrés. & $18 \%$ \\
\hline
\end{tabular}

Seulement $29 \%$ des consultants admettent qu'il est difficile de mesurer les résultats des interventions BSC et environ un quart pense que le BSC facilite la mise en place d'objectifs et de plans d'action. Il est difficile de mesurer les résultats du BSC, note un consultant, car « on obtient plutôt des résultats qualitatifs ». En effet, «les résultats les plus significatifs résultent $\mathrm{du}$ partage des impressions entre direction et employés sur la stratégie ».

En synthèse sur les résultats du BSC, on peut avancer que même si ceux-ci ne peuvent être chiffrés, ils sont significatifs aux yeux des consultants. On peut se demander cependant dans quelle mesure les résultats sont attribuables à l'outil $\mathrm{BSC}$, à son adéquation aux besoins du client ou bien encore à la manière dont il est mis en œuvre par le consultant.

Seulement $29 \%$ des consultants admettent qu'il est difficile de mesurer les résultats des interventions BSC et environ un quart pense que le BSC facilite la mise en place d'objectifs et de plans d'action.

\section{Pourquoi le BSC est-il moins utilisé en France?}

\section{Les différences culturelles entre la France et les pays anglo-saxons expliqueraient la sous-utilisation du BSC}

Parmi les 11 consultants sur 17 qui identifient les différences culturelles entre la France et les États-Unis comme étant un facteur de sous-utilisation du BSC, trois citent plus spécifiquement les différences culturelles liées à « la culture des résultats ». Les références aux différences culturelles sont très variées. Pour un consultant, « la culture latine française est très différente de la culture chiffrée et structurée américaine. On assiste en plus à une intellectualisation de la stratégie par les dirigeants en France. Ils pensent qu'à partir du moment où une stratégie a été définie, elle va naturellement se mettre en place. Cette mauvaise logique ne nécessite pas d'outils comme le BSC ». Pour un autre, l'écart d'idéologie se traduit par « une absence de sensibilité des Français pour le management $»$.

Sur un deuxième plan, beaucoup de consultants expriment l'idée que le BSC est un instrument de gestion trop «lourd » pour les entreprises françaises. Pour les uns, le BSC 
Tableau 5 : Principales raisons pouvant expliquer la sous-utilisation du Balanced Scorecard en France selon les consultants français en management

\begin{tabular}{|c|c|}
\hline Raisons & $\%$ \\
\hline $\begin{array}{l}\text { 1. Différence culturelle et idéologique entre la France et les pays anglo-saxons } \\
\text { concernant les pratiques de gestion. }\end{array}$ & $65 \%$ \\
\hline $\begin{array}{l}\text { 2. Modèle de management trop « lourd / dogmatique / structuré / procédurier / } \\
\text { formalisé / rationnel / théorique ». }\end{array}$ & $47 \%$ \\
\hline 3. Existence préalable de tableaux de bord de gestion en France et pas d'innovation du BSC. & $24 \%$ \\
\hline 4. Temps d’adaptation nécessaire. & $18 \%$ \\
\hline Mauvaise traduction du terme « Balanced Scorecard» en français et le terme fait peur. & $18 \%$ \\
\hline 6. Formation insuffisante des dirigeants français en management. & $18 \%$ \\
\hline $\begin{array}{l}\text { 7. Moindre recours au conseil externe en management en France et pas besoin } \\
\text { de consultants pour mettre en œuvre le BSC. }\end{array}$ & $18 \%$ \\
\hline
\end{tabular}

est « très formalisé et procédurier alors que les entreprises françaises encouragent la créativité et la liberté »; pour les autres, «c'est un modèle très dogmatique, à l'américaine ».

En troisième lieu, le BSC n'est pas jugé réellement novateur par rapport aux traditionnels tableaux de bord que les entreprises françaises utilisent. Un consultant va jusqu'à affirmer que «l'approche BSC est semblable à l'approche $\mathrm{ABC} / \mathrm{ABM}$ (Activity Based Costing/Activity Based Management). Elle n'apporte pas grand-chose de nouveau et peut être considérée comme ringarde au même titre que le concept de profit économique ».

En synthèse, on peut conclure que la sousutilisation du BSC est reconnue par les consultants. Ils l'attribuent principalement à des différences idéologiques entre les pratiques de gestion aux États-Unis et en France. Enfin, ils recommandent une utilisation plus « souple » du modèle, qui doit être adapté au contexte socioculturel des entreprises françaises.

\section{Les consultants sont plutôt optimistes sur le} développement du BSC en France
Les consultants sont plutôt optimistes sur le développement du BSC en France comme en fait foi le tableau 6 qui présente une synthèse des réponses les plus citées. Les deux tiers des consultants pensent que le BSC se développe ou peut se développer en France.

La sous-utilisation du BSC est reconnue par les consultants. Ils l'attribuent principalement à des différences idéologiques entre les pratiques de gestion aux États-Unis et en France.

Presque un tiers des consultants pense que le BSC n'est qu'un outil et que son utilisation ne se développera que si les besoins des clients l'exigent. Or, un consultant affirme que « depuis le début des années 90, le domaine de l'aide à la décision prend de l'importance en France » et une étude montre aussi que les démarches de gestion des performances en Europe se développent. Selon une étude ${ }^{8}$, $68 \%$ des entreprises européennes utilisent une démarche de gestion de la performance en 2003, dont la démarche BSC à $35 \%$. 
Cette tendance de fond concernant les pratiques de gestion des entreprises européennes et françaises laisse croire à un développement de l'utilisation du BSC en France. Un consultant

Tableau 6 : Développement de l'utilisation du Balanced Scorecard en France selon les consultants français en management

\begin{tabular}{|c|c|}
\hline Avis & $\%$ \\
\hline 1. L'utilisation du modèle se développe ou peut se développer en France. & $65 \%$ \\
\hline $\begin{array}{l}\text { 2. Le BSC n'est qu'un outil de management comme un autre et son développement } \\
\text { dépendra des besoins des clients. }\end{array}$ & $29 \%$ \\
\hline 3. Il n'y a pas de demandes et les cabinets ne développent pas d'offre spécifique au BSC. & $24 \%$ \\
\hline 4. Les entreprises peuvent le mettre en place seule ou bien sous un autre nom que BSC. & $18 \%$ \\
\hline $\begin{array}{l}\text { 5. Les grandes entreprises françaises doivent lancer le mouvement et des projets } \\
\text { d'envergure doivent faire leurs preuves en France. }\end{array}$ & $12 \%$ \\
\hline 6. Le BSC se développera avec la modernisation de l'État français. & $12 \%$ \\
\hline
\end{tabular}

remarque aussi que «le $\mathrm{BSC}$ touche à l'équilibre de la mesure de la performance, notion liée à la problématique du développement durable». Or, le développement durable est aussi un thème à la mode en Europe et en France depuis quelques années déjà. À ce propos, on peut noter que de nouveaux modèles de BSC intègrent un axe citoyen et tiennent compte du rôle social de l'entreprise'.

Un consultant note le développement accru des principes de la gouvernance d'entreprise en France depuis la fin des années 90. «On vient, en quelque sorte, par pression réglementaire aux systèmes de pilotage des organisations et au BSC en particulier. C'est une question de temps ». Un autre consultant remarque aussi « une évolution des pratiques vers plus de centres de responsabilités $(\mathrm{ABC} / \mathrm{ABM})$ qui devrait encourager le développement du BSC ». Selon un cabinet, le modèle BSC «devrait se développer, car il donne une représentation claire et pédagogique des facteurs de la performance d'une entreprise. De plus, il prolonge la démarche EFQM actuellement en vogue en France ».
L'European Foundation for Quality Management a pour mission de promouvoir le système de management de la qualité totale en Europe.

Trois consultants s'accordent pour dire que leurs clients peuvent mettre en place un BSC seuls, même sans le savoir. "La plupart du temps, les entreprises mettent en place le BSC de manière autonome, à partir de la littérature sur le sujet. Ils ne font appel à des cabinets que sur des points précis. Les entreprises françaises font peu appel au conseil externe, ce qui réduit le rôle des consultants dans la 'transposabilité' du modèle ».

Il est aussi possible d'évoquer la modernisation de l'État français comme facteur de développement de l'utilisation du BSC en France. «Depuis les lois organiques relatives aux lois de finances (LOLF) de 2001, les budgets sont votés par programme. C'est donc un changement réglementaire qui doit conduire à un changement dans la culture de l'État plus orienté vers la performance ». Votées en 2001, ces lois visent à moderniser la gestion publique et à renouveler la nature et les outils du contrôle parlementaire. Leur principal 
objectif est de passer d'une culture de moyens et d'une responsabilité de conformité à une culture et à une responsabilité de performance. En France, le ministère de la Défense a par exemple récemment mis en place un système de gestion inspiré du BSC.

En France, «le BSC n'est jamais mis en œuvre de manière globale », note un consultant. Selon un autre consultant, «pour que son usage se propage à toutes les entreprises françaises, il faut que des projets BSC d'envergure fassent leurs preuves dans les grandes entreprises. Or, ce n'est pas le cas pour l'instant $»$. Une autre explication est donnée par un consultant pour qui la démarche qualité associée au BSC n'est pas d'actualité pour les entreprises françaises concentrées sur la gestion des coûts. Pour lui, «la Chine serait le principal concurrent du BSC, car la pression que son modèle de production exerce sur les coûts détourne les firmes françaises du BSC et d'une gestion orientée sur la qualité »».
En synthèse, les avis sur le développement du BSC en France sont plutôt optimistes. Plusieurs courants de pensée relatifs à de nouvelles pratiques de gestion $\mathrm{y}$ font leur chemin. L'utilisation du BSC devrait se

développer. Les différentes fonctions du BSC peuvent rendre cet outil assez utile aux yeux des dirigeants d'entreprise. Cependant, une question rendra toujours difficile l'appréciation du degré d'utilisation du BSC. En effet, il est possible que certaines entreprises françaises utilisent la méthodologie BSC sans la nommer. Cette hypothèse, relevée par nombre de consultants, complique l'évaluation du taux d'utilisation du BSC par les entreprises françaises. Beaucoup d'organisations utiliseraient en effet la méthodologie BSC, mais sans la nommer ainsi.

En synthèse, les avis sur le développement du BSC en France sont plutôt optimistes. Plusieurs courants de pensée relatifs à de nouvelles pratiques de gestion $y$ font leur chemin.

\section{Conclusion}

On peut tirer quatre conclusions principales des résultats de l'enquête.

L'utilisation du BSC est réelle en France puisque presque la moitié des cabinets de conseil en management a au moins une expérience de mise en œuvre du BSC. La renommée du BSC est elle aussi large puisque les deux tiers des cabinets contactés connaissent le BSC.

Le BSC est perçu comme un outil d'alignement stratégique qui doit être utilisé lorsqu'il est adapté aux besoins du client et non un système global de management permettant de transformer toute une organisation en «entreprise orientée stratégie», pour reprendre 1'expression de Kaplan et Norton.

Les consultants sont assez optimistes sur l'utilité du BSC pour les entreprises françaises. Son développement en France devrait aller de pair avec celui de la généralisation des pratiques de gestion anglo-saxonnes et certains courants de pensée comme la gouvernance d'entreprise, la modernisation de l'État français, le développement durable, les démarches $\mathrm{ABC} / \mathrm{ABM}$ et la démarche EFQM.

La sous-utilisation actuelle du BSC en France semble être davantage due à un temps d'adaptation qu'à des difficultés de « transposabilité » du modèle aux entreprises 
françaises. Il doit toutefois être adapté au contexte culturel français. Parmi les adaptations soulignées dans cette étude, on note l'importance d'une démarche participative, l'utilisation plus souple des quatre axes et l'absence d'une double boucle de rétroaction.

Les résultats de l'enquête sur les différences culturelles entre la France et les États-Unis conduisent à se poser une question qui revient souvent à l'heure de la mondialisation : existe-t-il une exception culturelle française en matière de systèmes de gestion de la performance?

La réponse semble être positive, particulièrement en ce qui concerne le BSC. D'une part, moins de la moitié des consultants en management met en place des BSC en 2005. Les consultants admettent, de plus, qu'ils doivent adapter ce modèle à la culture des entreprises françaises pour le rendre efficace. D'autre part, seulement $3 \%$ des entreprises françaises pensent mettre en place un BSC. On peut cependant faire confiance aux entreprises françaises pour s'adapter à l'environnement mondial de plus en plus concurrentiel et pour utiliser progressivement des systèmes de pilotage stratégiques. Qu'ils se nomment BSC ou bien tableau de bord de gestion, les entreprises françaises ont sans doute déjà «transposé » les principes du BSC à leur organisation.

Malgré certaines limites dues à la méthodologie particulière de l'étude ainsi qu'aux caractéristiques de l'échantillon, cette enquête ouvre la voie à de futures recherches sur le sujet du BSC en France. Tout d'abord, celleci a révélé une grande diversité de points de vue de la part des consultants. Le but de l'enquête était d'explorer le thème de l'utilisation du BSC par les consultants français. Nous avons donc proposé des grilles d'analyse des réponses a posteriori et posé des questions très générales.

\section{Qu'ils se nomment BSC ou bien tableau de bord de gestion, les entreprises françaises ont sans doute déjà « transposé » les principes du BSC à leur organisation.}

Des questions plus détaillées permettaient d'établir des corrélations entre les adaptations du BSC, les phases de mise en œuvre et les résultats. Sur ce point, il serait intéressant de rassembler des études de cas d'entreprises françaises qui ont eu recours à des consultants pour mettre en œuvre un BSC. On pourrait alors estimer les avantages tirés de la mise en place du BSC.

Enfin, nous avons choisi à dessein un angle d'attaque du sujet BSC qui s'appuie sur les expériences des consultants. Il serait très intéressant d'élargir cette population cible et de poursuivre l'étude auprès des entreprises françaises à une échelle nationale. À une échelle internationale, il serait très pertinent de collaborer avec les chercheurs Lawson, Stratton et Hatch qui mènent actuellement une étude sur l'utilité, la conception et la mise en œuvre des BSC dans le monde entier.

\section{Bibliographie}

1 GEHRKE, I., HORVATH, P., 2002, «Implementation of performance measurement: a comparative study of French and German organizations », dans Epstein, M.J., Manzoni, J.F., « Performance Measurement and Management Control : A Compendium of Research », Studies in Financial and Management Accounting, vol. 9, p. 159-180;

ITTNER, C., LARCKER, D., 1998, « Innovations in performance measurement : Trends and research implications », Journal of Management Accounting Research, vol. 10, p. 205-238.

2 MALMI, T., 2001, « Balanced Scorecards in Finnish Companies: A Research Note », Management Accounting Research, vol. 1, n², p. 207-220.

3 Ax, C., BJornenaK, T., 2000, « The bundling and diffusion of management accounting innovations: The case of the balanced scorecard in Scandinavia », Proceedings of the 23rd Annual Congress of the 
European Accounting Association, Munich, Germany, 29-31 Mars;

WEGMANN, G., 2000, « Les tableaux de bord stratégiques : analyse comparative d'un modèle nord-américain et d'un modèle suédois », Gestion $2000, n^{\circ} 1$.

4 Bourguignon, A., Malleret, V., Norreklit, H., 2002, « L'irréductible dimension culturelle des instruments de gestion : l'exemple du tableau de bord et du balanced scorecard », Comptabilité-ContrôleAudit, vol. 8, $\mathrm{n}^{\circ}$ 1, p. 7-60.

5 HugOt, J.B., 2003, Guide des cabinets de conseil en management, Édition du management, l'Expansion.

6 KAPLAN, R.S.; NORTON, D.P., 2001, Comment utiliser le tableau de bord prospectif, Éditions d'organisation;
KAPLAN, R.S.; NORTON, D.P., 1998, Le tableau de bord prospectif, Éditions d'organisation.

7 LAWSON, R., StratTON, W., HATCH, T., 2004, "L'importance d'un véritable équilibre », «Automatisation du tableau de bord», "La clé du succès d'un système de tableau de bord », CMA Management, nos janvier, février et mars;

LAWSON, R., Stratton, W., HATCH, T., 2003, «Les avantages d'un système de tableau de bord ", "Stratégies gagnantes », "L'avantage tactique », CMA Management, nos juin-juillet, novembre et septembre.

8 PÉtRISSANT, A., 2003, «Pilotage d'entreprise : constat sur l'engagement des entreprises européennes en 2003 », IDC France.

9 NIVEN, P., 2003, Balanced Scorecard Step-By-Step for Government and Nonprofit Agencies, Éditions John Wiley \& Sons. 